\title{
Rationality Analysis of High-rise Building Structure Calculation un- der SATWE Procedure
}

\author{
(Hunan Jinding Architectural Design and Research Institute Chief Engineer, Shaoyang City, Hunan Province, 422000)
}

\section{Introduction}

In the high-rise building design process, due to building function, building modeling, planning and other factors, so that the structure of high-rise buildings increasingly complex. To solve the complex of high-rise buildings in the structural design of the problems, so that the structure design more reasonable and more economical and economical. In the high-rise building structure design, the SATWE software is used correctly, the design parameters and seismic force parameters are correctly input, the calculation results are correctly analyzed, and the structural model is modified according to the calculated results. The structural mechanics model is closer to the actual structure force the situation, and ultimately find a reliable program to calculate the results as a basis for structural design reinforcement map. In the process of analyzing the results of the calculation of the results of the calculation, the familiarity of the relevant specifications, the rationality of the high-rise building structure model, the accuracy of the input program parameters and the reliability of the program calculation results are the basic requirements of the high-rise building structure design.

We are in the structural design of the general steps in accordance with the following steps to calculate: structural model to establish (modify) $\rightarrow$ design parameters input $\rightarrow$ PKPM calculation $\rightarrow$ results output (self-vibration cycle to determine the vibration curve, seismic force to determine the displacement angle The judgment of the axial compression ratio, the analysis of the problem, the judgment of the process integrity, the final design of the process.

According to the above steps in strict order, the following will be a detailed analysis of the results of the computer.

2 To determine the rationality of the calculation, control and adjustment

\subsection{Self-oscillation period}

The vibration period is an important parameter of structural design, especially in the earthquake-resistant area is more important. If the input of the vibration period is not accurate, the calculation structure is not reliable. The self-vibration period of the structure is closely related to the stiffness of the building structure itself. The smaller the cycle, the smaller the smaller the cycle.

The self-oscillation period of the initial calculation is given in the following range:

Frame structure $\mathrm{T} 1=(0.08 \sim$ $0.1) n$

Frame - shear wall structure T1 $=(0.06 .0 .08) \mathrm{n}$

Shear wall structure $\mathrm{T} 1=0.04$ $\sim 0.05)>n$

$\mathrm{T} 2=(1 / 3 \sim 1 / 5) \mathrm{T} 1, \mathrm{~T} 3=(1 / 5$ 1/7) T1

$T 1, T 2, T 3$, respectively, for the first cycle, the second cycle, the third phase; $\mathrm{n}$ for the number of layers.

\begin{abstract}
In recent years, with the increasing shortage of land resources in our country, in order to make full use of limited land resources, the development of construction industry is developing towards high-rise and super-high-rise buildings. This is based on the use of structural design and structural calculation of building structures with the higher requirement. For structural designers, the structural model is determined reasonably, the structural design procedure is used correctly, the design parameters and seismic force parameters are accurately input, the results are correctly analyzed and the structural calculation model is adjusted according to the calculated results. Repeated adjustments in order to design safe high-rise buildings, so that the capital construction investment has been rationally used. In this paper, how to use the SATWE program developed by China Academy of Building Science to study the structural calculation and structural problems, how to analyze the calculation results and use the calculated results to reflect the problem and then adjust the structural model and other issues to study.
\end{abstract}

Keywords: high-rise building structure, SATWE program, calculation result

Published online: 15th July, 2017

Calculate and adjust the results of the calculation points: 
(1) If the calculation result deviates from the above range, it is necessary to check whether the body conforms to the requirement of simple, symmetrical and balanced, and adjust the layout and facade shape according to the specification, and then recalculate until the calculation result The above range is close. Under normal circumstances, for the structure of a simple, uniform, symmetrical results to adjust 2-3 times to meet the requirements.

(2) Calculate the period using the mode decomposition response spectrum method. For methods with seismic fortification requirements, this method is a method commonly used to calculate the structural self-oscillation period, but attention should be paid to the determination of the model and the number of modes (as described in the "SATWE User Manual" and "PKPM Xintiandi").

(3) The calculation of the results of the cycle is more complex, the first thing to do is to find the cause of the problem, adjust the structure of the layout, the balance of the structural stiffness of the complex design for the first high-level design of the design staff need to draw on similar projects design experience and learning experience to shorten the time to solve the problem.

\subsection{Vibration curve, seismic force,}

Mode curve: Under normal circumstances, if the structure is reasonable, then the selected vibration curve is continuous, the middle generally does not appear breakpoint or obvious bending.

In general, there is no zero in the first mode; the second mode has a zero point at $(0.7 \sim 0.8) \mathrm{H}$. The third mode has two zero points at $(0.4 \sim$ $0.5) \mathrm{H}$ and $(0.8 \sim 0.9) \mathrm{H}$, respectively,

Seismic force: The second force of the earthquake is Newton's second theorem, which is related to the mass and the acceleration of the earthquake. After the structural model is built, the quality is determined. Therefore, it is important to input the seismic acceleration correctly after the structural model is completed. It is related to the damping ratio of the structure, the type of the site where the structure is located, the intensity of the earthquake, and the magnitude of the seismic fortification to 6 degrees.

From the physical principles we can see that the greater the rigidity of the building structure, the more severe the destruction of the earthquake, so we have to adjust the structure according to the structure of stiffness, so that the uniformity of stiffness, to avoid the individual component stiffness is too large and in the earthquake under the first destruction, resulting in the other components were broken.

\subsection{Displacement angle (ratio)}

Displacement ratio: The ratio of the maximum horizontal displacement between the floors and the floor level.

The elastic displacement angle of the layer is the ratio of the elastic displacement and the height of the building floor, which is the control index of the weak structure.

The SATWE program in the PKPM software outputs the WDISP.OUT file to the horizontal displacement, the lateral displacement, the average displacement level, and the displacement angle and the corresponding ratio of the building floor. According to the results of the displacement angle to note the following:

(1) If the ratio of lateral displacement to average displacement is more than half, it should be checked whether the parameters of the upper layer are accurate and sometimes the effect of bi-directional vibration is taken into account.

(2) The displacement calculated by this method also takes into account the effects of accidental factors, and the interlaminar displacement angle does not need to take these factors into account.

2.4 Axial compression ratio, shear weight ratio, stiffness ratio, stiffness ratio

Axial Compression Ratio: Column (Wall) Axial Compression Ratio The value of the column shaft pressure is proportional to the cross-sectional area of the column (wall) and the flexural compressive strength of the reinforced concrete.

Shear weight ratio: the minimum seismic force of the floor of the shear force coefficient, high-rise building structure to pay attention to the structure is weak, the weak structure of the floor, should be multiplied by 1.15 magnification factor.

Calculate and adjust the results of the calculation points:

(1) The designer of the unreasonable floor calculation results to find the reasons, modify the model, to ensure that the floor structure of the minimum seismic shear coefficient within the allowable range.

(2) The seismic force of each floor should be consistent with the design parameters in the floor; if the user to consider the automatic amplification, will enlarge SATWE WZQ. OUT input procedures used in the value.

The ratio of the lateral stiffness to the design value of the gravity load in the high - rise structure.

Calculate and adjust the results of the calculation points:

(1) Calculate the equivalent lateral stiffness according to the formula.

(2) For the high-rise building frame structure, when the stiffness ratio is greater than 10 , when the highrise building structure design more reasonable and stable. When the stiffness ratio is greater than 20 , this time can ignore the impact of gravity on the high-rise building structure.

(3) For the curved shear wall structure, side structure, when the stiffness ratio is greater than 1.4 , the floor of the building structure is relatively stable; when the stiffness ratio greater than 2.5 , the second order effect of gravity on the floor building structure, which is not dif- 
ficult to be obvious, or even negligible.

Calculate and adjust the results of the calculation points:

(1) When calculating the stiffness ratio of the structural layer, it is necessary to calculate the displacement level, as in the assumption that the building floor is rigid. In the case of the rigid floor to determine the floor structure to find a relatively weak floor, and then continue to complete the calculation.

(2) Above the calculation of floor stiffness calculation method, the designer should be based on the actual construction to make a reasonable choice:

(3) 2. 5 structural rationality judgment

Relatively speaking, relatively normal building floor structure, there will not be too many standard layers, to follow the following building rules;

The design of the structure of the bottom column and the wall of the axial force for the pressure; column, wall mostly structural reinforcement; beam basically no super-tendons;

Calculated results as long as the above meet the requirements, it can be considered correct, you can apply the results of the calculation of high-rise building structure to go. It should be noted that the above requirements are summarized by some representative buildings, and for some complex high-rise buildings, the calculated results may somewhat deviate from the reality, which is inevitable. Designers as long as they can grasp the high-rise building structure of the relevant principles, encountered problems to more similar projects to learn from the successful experience to shorten the exploration time, designed to meet the use of functional requirements of the building products.

\section{Conclusion}

With the progress of the times, highrise buildings are constantly developing, because the high-rise building structure is more complex and therefore difficult to complete once, need to continue to adjust to ensure the rationality of high-rise building structure and the accuracy of the results. The relevant staff to learn from the past experience, the number of calculations, the results of each calculation to find out the reasons for the error, and then adjust the structural model, so that the structural model close to the actual mechanical model, through a time of the calculation, modify, so that the structural model again and again, and ultimately to be satisfied with the results. To pay more attention to the theoretical results of high-rise building structure, combined with practice, and constantly design a scientific and reasonable, safe and beautiful, comfortable and comfortable high-rise buildings, it is worth noting that the results of any problems are structural model problem data performance, adjust the structural model is fundamental, is the source, leaving the structural model to talk about parameters that are only water without a source, and a tree without roots.

\section{References}

[1] "High-Rise Building Concrete Structure Technical Regulations Application and Analysis" China Construction Industry Press, Zhu Bing Yan Edited

[2] Chen Tiewen. Study on Some Problems in the Design of Frame Structure of High-Rise Building Frame [J]. Building materials and decoration. 2016 (26)

[3] Hou Jie, Feng Yuan, Wu Shu, Liu Zhaqing, Kang Yongjun. SATWE READ Software Development and Application of [J]. Sichuan Architecture. 2013 (02)

[4] Xu Zhe, Tu Zhongyao, Qian Guozhen. Application of SATWE Software to Discuss a Number of Issues [J]. Zhejiang Architecture. 2013 (05)

[5] Jiao Yukun .2010 Version of SATWE the Main Calculation Parameters of the Correct Selection [J]. Project Construction and Design. 2012 (S1)

[6] "SATWE User Manual"

[7] "China Earthquake Parameters Zoning Map" (GB18306-2015)

[8] "Code for Seismic Design of Buildings" (GB50011-2010)

[9] "High-rise Building Concrete Structure Technical Regulations" (JGJ3-2010) 